\title{
Electrogenerated Chemiluminescence Detector for Flow Injection Analysis
}

\author{
Masanori SATo and Takeshi YAMADA \\ Laboratory of Analytical Chemistry, Faculty of Textile Science, \\ Kyoto Institute of Technology, Matsugasaki, Kyoto 606
}

\begin{abstract}
A new kind of detector system based on electrogenerated chemiluminescence (ECL) was devised for flow injection analysis. The flow through type of electrolytic cell was modified to observe the ECL. The quartz optical window was set in front of the working electrode (Pt plate). The observed luminescence intensity was amplified by using a lock-in amplification technique. The preliminary examination of the whole system was performed using luminol with a strong $\mathrm{ECL}$. The injection of a sample solution in a carrier solution of sodium carbonate caused a peak-shaped luminescence at the electrode. Next the determination was performed for non-luminescent compounds after the pre-labeling with luminol. It was found that oligo peptides or bovine serum albumin can be determined by ECL method. Though, the sensitivity and the reproducibility of ECL method are almost comparable to existing methods, it has the advantage that two kinds of quantities, the luminescence intensity and the electrolytic current, can be obtained at the same time.
\end{abstract}

Keywords Electrochemiluminescence, flow injection analysis, luminol, labeling reagent, oligo peptides

The recent advances of the flow analytical systems like flow injection analysis (FIA) or high performance liquid chromatography (HPLC) depend on the developement of the several kinds of detection systems. ${ }^{1-5}$ Among the spectroscopic methods of analysis, there is a detection method that determines the interested molecules using the chemiluminescence emitted by the electrode oxidation or reduction. The phenomenon, termed electrogenerated chemiluminescence (ECL), is known to be related to the chemically generated chemiluminescence. In general, the chemiluminescence spectrum of a molecule coincides with its fluorescence spectrum. It is meant that the excited species is participated in the course of the emission processes. The ECL spectrum of a molecule also coincides with the fluorescence spectrum of a chemical species which participated in the electrode reaction. Thus the advantage of the ECL detection system over the fluorometry or the chemiluminescence detection system is as follows. As an excitation light-source is not needed, there is no problems related to the light-source fluctuation or to the fluorescence of the solvent itself or impurities. Moreover, it is possible to simplify the FIA system, because the addition of oxidant is not required.

As for the ECL compounds, luminol (5-amino-2,3dihydro-1,4-phthalazinedione) has been used in many studies. ${ }^{8}$ The luminol anion in alkaline solution is known to be oxidized at the anode, and emits a strong luminescence in the presence of dissolved oxygen. Though the precise mechanism was studied by Kuwana and his coworkers ${ }^{9-11}$ using voltammetric methods, there are few studies on the analytical application of ECL. ${ }^{12,13}$ We have been studying the utility of ECL for the flow systems such as FIA ${ }^{14}$ and HPLC. This paper describes an ECL detector system for FIA. In addition, the determination of biologically interesting molecules was performed after the pre-labeling of these molecules with luminol.

\section{Experimental}

\section{Reagents}

The purchased reagents were of analytical-reagent grade. Luminol was stored in a light-tight desiccator after the conventional purification. ${ }^{9}$ Luminol solution was prepared by diluting $1 \mathrm{mM}$ luminol stock solution with $0.1 \mathrm{M}$ sodium carbonate solution.

Based on the results of the previous study ${ }^{14}, 0.1 \mathrm{M}$ sodium carbonate solution was used as a carrier solution at a flow rate of $0.8-1.2 \mathrm{ml} / \mathrm{min}$ throughout the experiments.

The derivatization of oligo peptides or proteins was performed according to the reported procedures ${ }^{15,16}$; after diazotization of the amino group of luminol, the coupling reaction of interested molecules, having the amino or imidazole group, with diazo-luminol was followed. 


\section{Apparatus}

The experimental setup for FIA is shown in Fig. 1. The reservoir contained $0.1 \mathrm{M}$ sodium carbonate solution as a carrier. A low pressure plunger pump (FMI Co. type RP-SY) supplied the carrier solution at a constant rate. The sample injector (Rheodyn Co.) made of Teflon had a constant loop capacity of 0.1 $\mathrm{cm}^{3}$.

The electrolytic cell for the ECL observation was designed in our laboratory. The schematic assembly of the cell is represented in Fig. 2. The main body of the cell was composed of two pieces of Diflon square blocks tightly fixed to each other with a Teflon sheet spacer (cell volume; ca. $0.02 \mathrm{~cm}^{3}$ ). As for the potentiostatic control of the electrolytic system, a three-electrode device was used; the working electrode

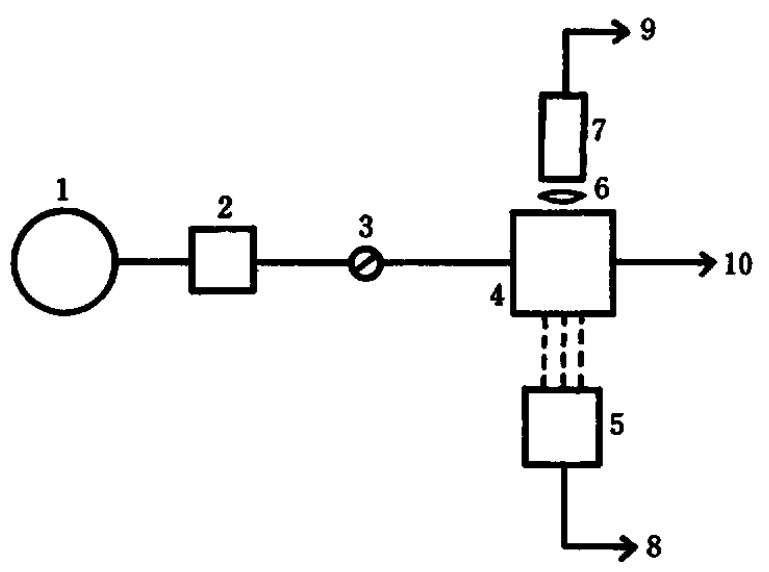

Fig. 1 Experimental setup for the ECL. 1, reservoir; 2, pump; 3, injector; 4, ECL cell; 5, potentiostat; 6, lens; 7, photomultiplier tube; 8, current; 9, luminescence; 10, waste.

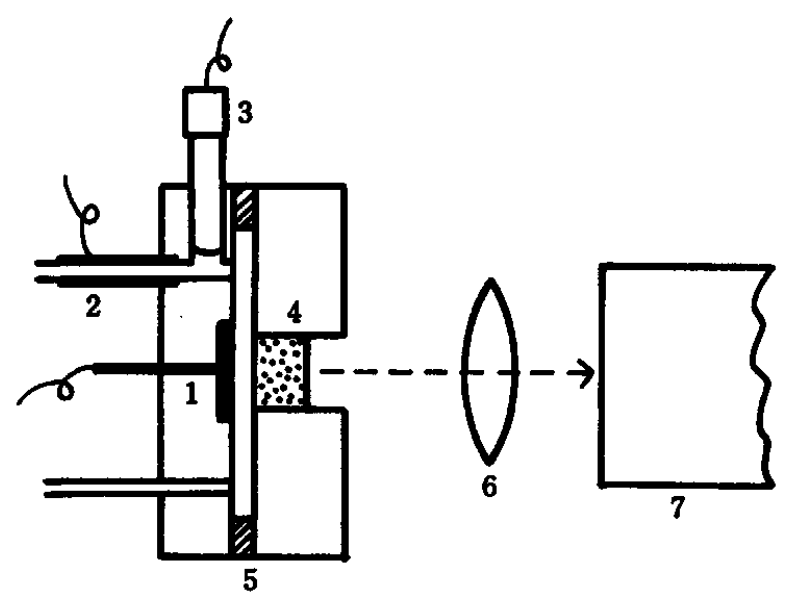

Fig. 2 ECL cell. 1, working electrode; 2, counter electrode; 3 , reference electrode; 4 , quartz window; 5 , Teflon spacer; 6 , lens; 7 , photomultiplier tube. was a bright platinum plate $\left(5 \mathrm{~mm}^{2}\right)$, the counter electrode at the outlet consisted of a stainless steel pipe, and the reference electrode was $\mathrm{Ag} / \mathrm{AgCl}$ with $1 \mathrm{M}$ potassium chloride solution.

The potentiostat (Prinston Appl. Res., Co. type 174A) was used for both the voltammetric study with the potential programmer and the FIA at a constant potential. In the FIA study, a two-pen Y-T recorder (National Co. type VP-6537A) was used for the recording of the electrolytic current as well as the luminescence intensity.

The cylindrical quartz window was set in front of the working electrode to observe the total emission intensity. The light beam was focused on the photomultiplier tube by using a set of combinated lenses. When the luminescence intensity was not weak, the photomultiplier current was directly amplified without the aid of the lock-in amplification technique.

\section{Results and Discussion}

\section{Effect of flow rate}

Typical recording in FIA is shown in Fig. 3. The luminescence intensity showed a peak-shaped curve that corresponded to the electrolytic current. With the increase in flow rate, the electrolytic current gradually increased, whereas the luminescence intensity rather decreased at a high flow rate. This is probably due to

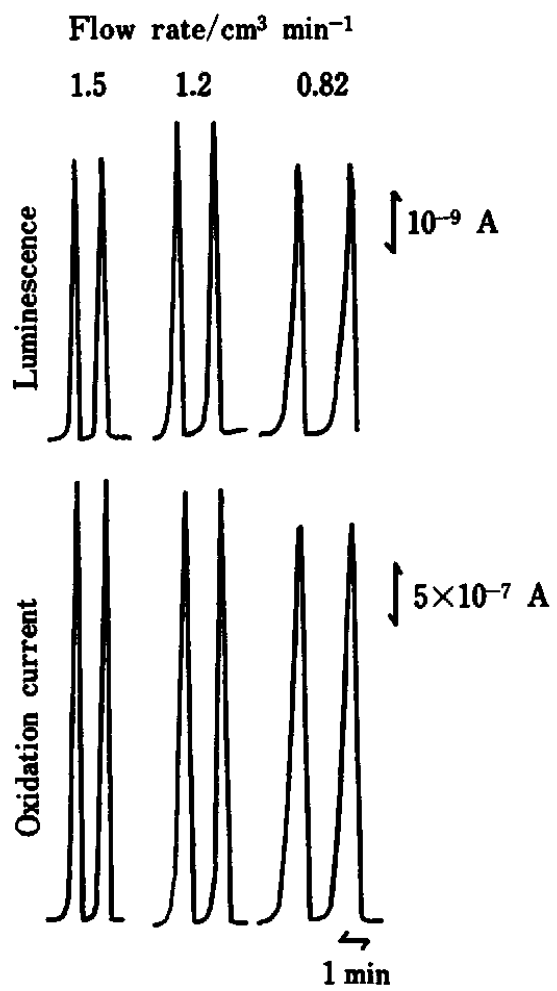

Fig. 3 Effect of flow rate. $10^{-4} \mathrm{M}$ luminol; $0.1 \mathrm{M}$ sodium carbonate solution. 
the fact that excited intermediate ions were formed as a result of the secondary chemical reaction after the electrode oxidation. ${ }^{10}$

\section{Hydrodynamic voltammogram and luminescence}

Figure 4 shows the hydrodynamic voltammogram $A$ and the luminescence-potential curve $B$ of luminol. The bell-shaped curve seemed unusual, because the

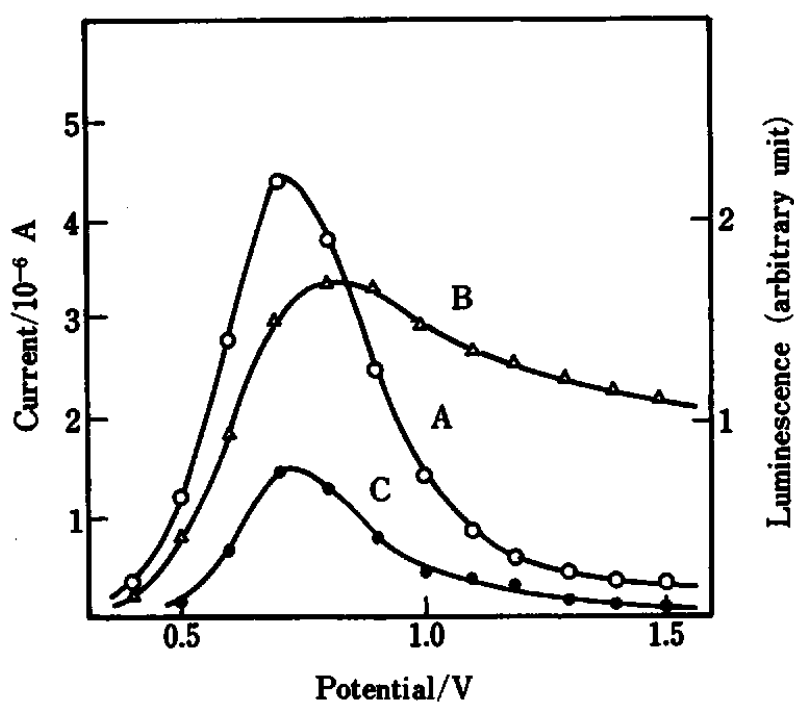

Fig. 4 Hydrodynamic voltammogram and luminescencepotential relationship. $\mathrm{A}, 10^{-4} \mathrm{M}$ luminol (current); $\mathrm{B}$, $10^{-4} \mathrm{M}$ luminol (luminescence); $\mathrm{C}, 10^{-4} \mathrm{M}$ phthalhydrazide (current); solution, $0.1 \mathrm{M}$ sodium carbonate.

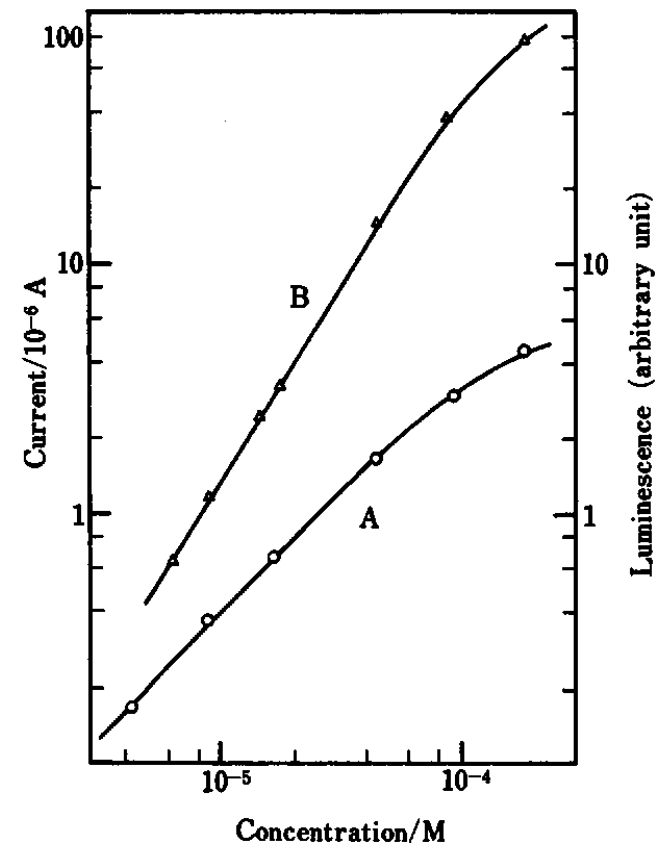

Fig. 5 Calibration curve for luminol. A, electrolytic current; B, luminescence intensity. ordinary hydrodynamic voltammogram shows the Sshaped curve similar to an ordinary polarogram. This may be attributed to the instability of the electrode surface at the potential region where the oxygen production begins to occur. The hydrodynamic voltammogram $\mathrm{C}$ of phthalhydrazide, an analogue of luminol, also showed similar behavior with luminol. This fact suggests that the amino group of luminol is not the reason for the abnormality. The luminescence curve B showed parallel behavior with $\mathrm{A}$. The disturbed oxidation of luminol is also suggested by this phenomenon. Based on these results, the electrode potential was kept constant at $+0.7 \mathrm{~V}$ throughout the experiments.

\section{Calibration curve for luminol}

The calibration curves for both the electrolytic current and the luminescence intensity are shown in Fig. 5. The luminescence curve had a steeper inclination than that of the electrolytic current. Thus, the luminescence was more sensitive than the electrolytic current at a rather high concentration range. The detection limit for luminol was about $10^{-9} \mathrm{~mol}$. Though the sensitivity was not so high in this case, it has the advantage that a baseline of the emission signal is more stabilized than that of the electrolytic current because of the lock-in amplification of the luminescence intensity. The reproducibility of the determination at $10^{-5} \mathrm{M}$ was $3 \%$.

\section{Effect of other compounds}

The effect of other coexisting compounds on the ECL of luminol was examined and represented as a relative value in Table 1 . Contrary to the case of chemiluminescence by an oxidant, the inorganic ions such as iron and cobalt had no significant effect on either the electrolytic current or the luminescence intensity. However, ascorbic acid had a considerable effect, which is probably due to the bulk chemical reaction with luminol even without the electrode oxidation.

\section{ECL of luminol derivatives}

As an analytical application of ECL, the derivatization of non-chemiluminescent compounds with diazoluminol was investigated. One typical example is a triglycine derivative of luminol. As shown in Fig. 6, the cyclic voltammogram of this compound has one

Table 1 Effect of coexisting compounds on luminol

\begin{tabular}{lcc}
\hline \multirow{2}{*}{ Compounds } & \multicolumn{2}{c}{ Relative ratio } \\
\cline { 2 - 3 } & Current & Luminescence \\
\hline Luminol $\left(0.84 \times 10^{-4} \mathrm{M}\right)$ & 1.0 & 1.0 \\
Tyrosine $\left(0.70 \times 10^{-4} \mathrm{M}\right)$ & 1.07 & 0.83 \\
Ascorbic acid $\left(1.7 \times 10^{-4} \mathrm{M}\right)$ & 1.10 & 1.22 \\
$\mathrm{~K}_{3} \mathrm{Fe}(\mathrm{CN})_{6}\left(0.97 \times 10^{-4} \mathrm{M}\right)$ & 1.06 & 1.01 \\
$\mathrm{Co}\left(\mathrm{NH}_{3}\right)_{6} \mathrm{Cl}_{3}\left(0.97 \times 10^{-4} \mathrm{M}\right)$ & 1.04 & 0.98 \\
\hline
\end{tabular}


oxidation wave that corresponded to the first wave of luminol. The absence of the second wave for this derivative can be attributed to the absence of the amino group as a result of the coupling reaction. The calibration curve of the derivative is shown in Fig. 7. The concentration range suitable for the determination is comparable to that of free luminol. The reproducibility of the determination at $10^{-5} \mathrm{M}$ was $3 \%$.

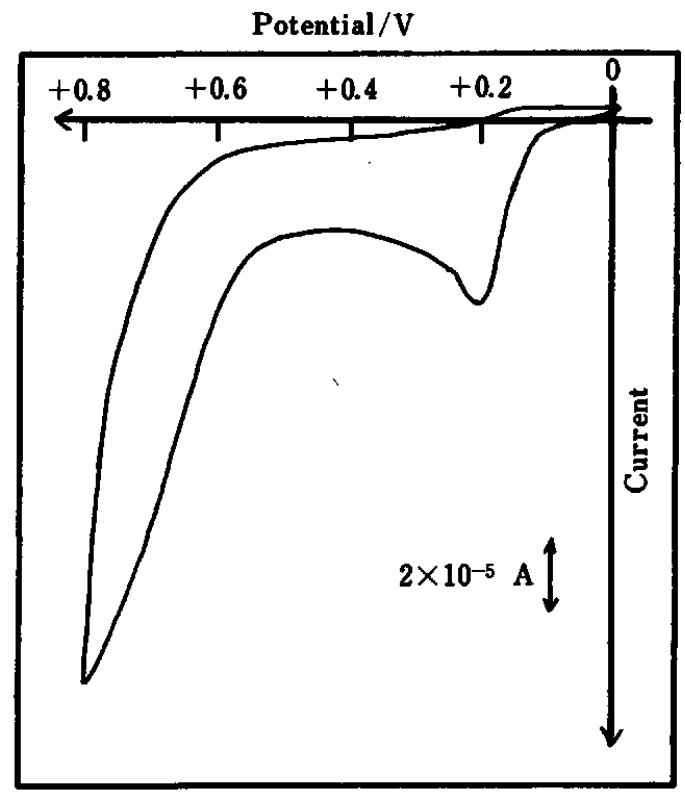

Fig. 6 Cyclic voltammogram of luminol-Gly-Gly-Gly. Concentration, $10^{-4} \mathrm{M}$; solution, $0.1 \mathrm{M}$ sodium carbonate; potential scan rate, $0.02 \mathrm{~V} / \mathrm{s}$; working electrode, glassy carbon disk $\left(0.19 \mathrm{~cm}^{3}\right)$.

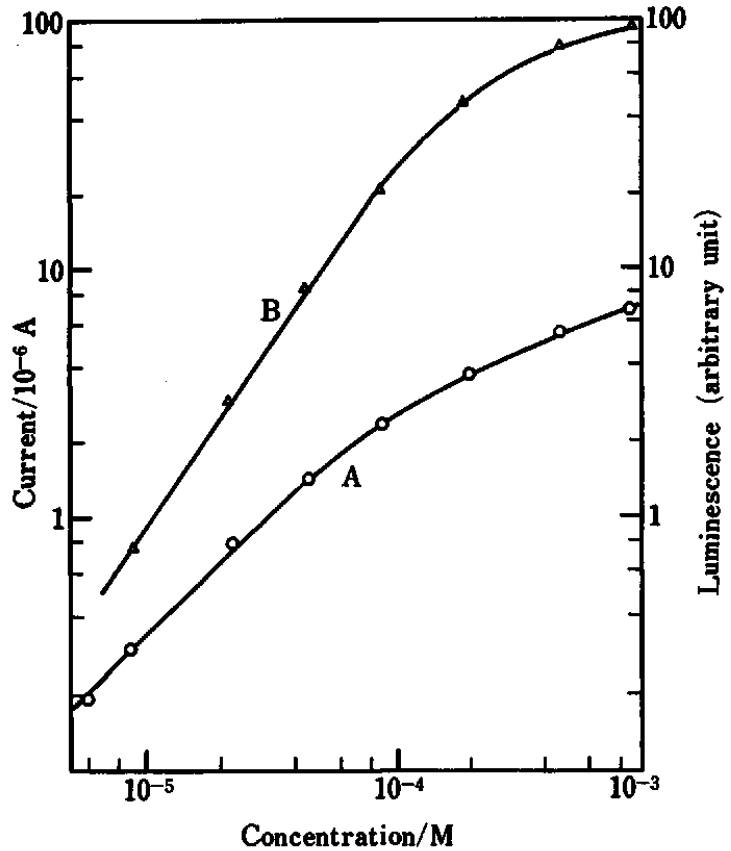

Fig. 7 Calibration curve for luminol-Gly-Gly-Gly. electrolytic current; B, luminescence intensity.

\section{Luminescence efficiency of derivatives}

Table 2 shows the relative luminescence efficiency of some derivative compounds refered to free luminol. At the same concentration range as luminol, the emission efficiency of the derivatives diminished by the diazocoupling reaction, but is still much better than that of free isoluminol.

\section{Luminol derivative of $B S A$}

As a model procedure for the determination of proteins by ECL, bovine serum albumin (BSA) was coupled with diazo-luminol according to the reported procedures. ${ }^{15,16}$ The calibration curve of the derivative is shown in Fig. 8. Although the coupled number of luminol per one molecule of BSA is not exactly known, the luminescence intensity was linearly dependent on the concentration of BSA.

The result of this experiment revealed that a more favorable procedure of derivatization is required to improve further the sensitivity of the determination for derivatized molecules having high molecular weights.

In conclusion, we found that the ECL detector has sufficient utility as a detection system in FIA. The results of preliminary experiments using luminol as a chemiluminescent compound revealed that the sensitivity and the reproducibility of ECL system are comparable to the other methods. However, the

Table 2 Luminescence efficiency of derivatives

\begin{tabular}{lc}
\hline \multicolumn{1}{c}{ Compounds } & $\begin{array}{c}\text { Relative ratio } \\
\text { (luminescence/current) }\end{array}$ \\
\hline Luminol & 1.0 \\
L-Gly-Gly-Gly & 0.72 \\
L-Tyr & 0.30 \\
L-Tyr-Gly & 0.17 \\
L-Tyr-Ala & 0.14 \\
L-His-Gly & 0.25 \\
Isoluminol & 0.0079 \\
\hline
\end{tabular}

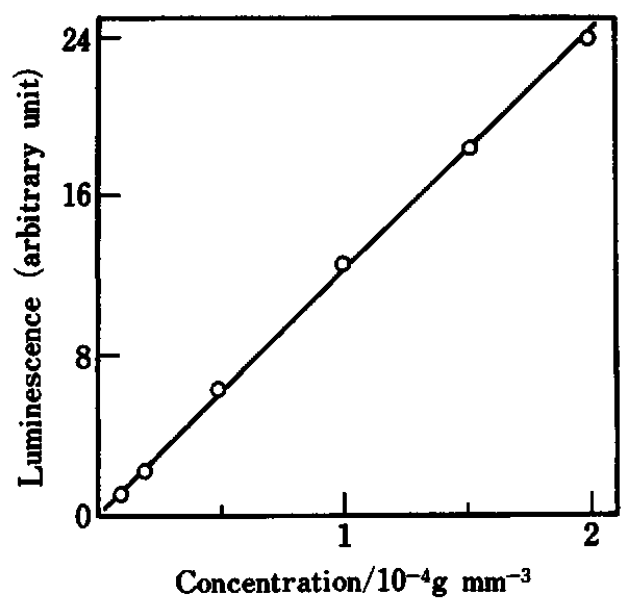

Fig. 8 Calibration curve for BSA derivative. 
advantage of ECL detection is that the system does not require an exciting light-source as in the case of fluorescence detection. Therefore, several problems arising in the fluorometric determination can be avoided. Furthermore, it is possible to simplify the FIA system compared with the case of the chemiluminescence detection system in which the addition of oxidant or catalyst is indispensable.

One of the useful application was found to be the determination of non-luminescent compounds after pre-labeling with luminol. It was possible to determine some oligo peptides and proteins by ECL detection in FIA after the coupling reaction with diazo-luminol. As for the problem of increasing the sensitivity, further investigation is needed to find another type of coupling reaction and to synthesize new compounds more suitable for the ECL determination.

The another advantage of the ECL system is that the simultaneous observation of the electrolytic current and the luminescence intensity can be performed with the same detection cell. This feature will be advantageous for the selective determination as in HPLC. In fact, the ECL cell used in FIA serves as a detector in HPLC without any modification. We are now investigating the applicability of the ECL detection system in HPLC.

\section{References}

1. T. M. Freeman and W. R. Seitz, Anal. Chem., 50, 1242 (1978).
2. J. L. Burguera, A. Townshend and S. Greenfield, 'Anal. Chim. Acta, 114, 209 (1980).

3. K. Honda, J. Sekino and K. Imai, Anal. Chem., 55, 940 (1983).

4. M. Tabata, C. Fukunaga, M. Ohyabu and T. Murachi, J. Appl. Biochem., 6, 251 (1984).

5. W. R. Heineman and H. B. Halsall, Anal. Chem., 57, 1321 A (1985).

6. L. R. Faulkner and A. J. Bard, "Electroanalytical Chemistry", Vol. 10, p. 1, ed. A. J. Bard, Marcel Dekker, New York (1977).

7. M. Sato, Rev. Polarogr. [Kyoto], 19, 29 (1973).

8. O. H. Hercules, "Technique of Chemistry", Vol. 1, p. 275, Wiley, New York (1971).

9. E. T. Seo and T. Kuwana, J. Electroanal. Chem., 6, 417 (1963).

10. B. Epstein and T. Kuwana, Photochem. Photobiol., 4, 1157 (1965).

11. B. Epstein and T. Kuwana, Photochem. Photobiol., 6,605 (1967).

12. M. Sato and T. Yamada, "Trace Analysis" (in Japanese), p. 63, ed. T. Fujinaga and S. Ikeda, Kagaku-Dojin, Kyoto (1978).

13. M. Sato, T. Yamada and M. Fujino, Nippon Kagaku Kaishi, 1981, 74.

14. M. Sato, T. Yamada and M. Horikawa, Denki Kagaku, 51, 111 (1983).

15. J. S. A. Simpson, A. K. Campbell, M. E. T. Ryall and J. S. Woodhead, Nature [London], 279, 646 (1979).

16. E. Konishi, S. Iwasa, K. Kondo and M. Hori, J. Clin. Microbiol., 12, 140 (1980).

(Received September 26, 1986)

(Accepted September 29, 1986) 